\title{
Video Conference Application using Android and Firebase
}

\section{Rahul Roy ${ }^{1} \mid$ SeemaKalonia $^{2}$}

1 IT (Information Technology), Maharaja Agrasen Institute of Technology,

2 Professor, IT (Information Technology), Maharaja AgrasenInstitute of Technology,

\section{To Cite this Article}

Rahul Roy and SeemaKalonia, "Video Conference Application using Android and Firebase", International Journal for Modern Trends in Science and Technology, 6(12): 463-465, 2020.

\section{Article Info}

Received on 16-November-2020, Revised on 09-December-2020, Accepted on 12-December-2020, Published on 19-December-2020.

\section{ABSTRACT}

As the world is changing rapidly and there has been a significant increase in the number of technologies which are getting introduced over the years to bridge the gap between the people. Video conferencing is one of the that which has seen a significant growth over the years. Understanding what are required for video conferencing and its application has become one amongst the foremost important researched topics by various learning institutions and businessmen. In this paper, an introduction to video conferencing is presented with the strain on its application in distance learning.

KEYWORDS:Video Conferencing, Androidapplication, Video call

\section{INTRODUCTION}

Video Conferencing has emerged the modern technology in a new era. This is the only technology that is usable, stable, and affordable in this modern time. The video has been proved to be the next revolution in the electronic world. Many companies are involved in developing a system to support Virtual technology, telecommunication and remote conferencing. Video conferencing has become the easiest of communicating person over a large geographical Area. Also the money involved in this communication is the cheapest across digital communication. Todays the technology involved in building a video conference application has made it so advanced in terms of audio and video by using various technologies such that the functioning of the application of a band is smooth. Accessories involved in a web conferencing application such as web cam, microphone is much cheaper, thus allows anyone to participate in a video conference.
The software and the hardware application used in this process is available easily today. The lower budget and gaining, visualizing multiple knowledge, locations diversity all over the world has been made possible due to the video application, where the modern institutions are adopting practices on low budget. The participants over an video application use hardware accessories. The modern video applications not only allows video conference but also provide multiple features like image sharing, file sharing, and also communicate over text. Due to the management of bandwidth over same meeting, the number of persons joining a meeting is rapidly growing. The video conference limited to one to one talk has also been part of e-learning, classroom learning etc. In Education field using this technology the educators now feel more expressive in delivering thoughts, also a bidirectional communication is possible over this technology. 


\section{VIDEO CONFERENCING}

Video conferencing is a way of communicating between two persons, over a physical medium where they share their audio, video are share over by converting these in digital signals and then transmitting over a medium. The video application allows a person to share not only his thoughts to a person, but also the expressions are also share and communicated. The application uses various hardware and software technologies. A video conference is connected at two or more locations using video application. The communication over two persons or more can happen over a home, studio, office anywhere via the hardware application that provides us the facility to join over that conference. The modern tech video applications are supported over low graded phones, 3rd generation phones, laptops, etc. These applications are also supported on a device that lacks the modern world disciplinarians. Besides all the audio and video these applications are designed to share texts, files and other stuffs designed and displayed on whiteboard

2.1. Components of Video Conference Videoconferencing has three essential components:

- The Hardware.

- The intermediate network that carries the signals between sites.

- The conference environment or room.

2.1.1. The Hardware Basic equipment required for a video conference session include a camera, microphone, a video conferencing unit, display unit, and audio system (JNT Association, 2007; Alan, 2009). Camera - A device used to capture images and convert them into an electrical signal. Location of the camera must be ideal to permit for realistic eye-contact. Also, good quality and functionality of the cameras should be ready to provide a sharper, more colorful image, with less visual noise. Microphone-Microphones utilized in $\mathrm{VC}$ are usually very sensitive and will be placed faraway from equipment's like projectors which may produce some ground noise. Video Conferencing Unit - The VC unit usually referred because the codec coder / Decoder) accepts the vision and sound signals (video and audio) and processes them into a acceptable format for transmission through the network to the remote site. The Decoder does the reverse at the receiving end: it accepts the digital signals from the remote site over the network and decodes or converts these into video and audio. Eventually this audio and video are fed to a display unit and speaker to display the images and reproduce the sound from the remote site respectively. Display Unit - A display unit are often either a TV unit or a projector projecting onto a surface. The display unit is connected onto the codec. Audio System - an honest sound system is right for video conferencing. Sometimes, TV speakers are used but usually (i.e. classroom, boardrooms, etc.), an honest sound system with mixer, amplifier and speakers could be required.

\subsection{2.}

Today, the Network Video conferencing technology is working across different internet protocol (IP) networks and integrated system digital Network (ISDN). With these vast networks, videoconferencing has the capability for connecting worldwide audiences. With IP transmission, the results are often variable because the videoconference data has got to compete with other computing data. ISDN guarantees connections at the chosen quality, giving more reliable conferences, but as call charges are levied it's also costlier than IP. A simple video conference are often initiated with as low as $384 \mathrm{kbps}$ with 30 frames of video per second real-time.

2.1.3. The Conference Environment Lighting is a simple thanks to improve picture quality. If the space isn't specially built or equipped for video Conferencing, it's probable that there aren't enough lights to supply the optimum quality for the video Conference cameras. The result's a flickering visual noise seen especially when the cameras are zoomed in (Sami, 2008). Another result is a lack of color saturation. Thus proper lightning is a simple thanks to improve video quality. Also, the space should be acoustically designed to avoid the echo.

2.2. Benefits of Video Conferencing

- Sharing of presentations

- It allows instant, full-duplex two way communication of content; verbal, pictorial objects etc.

- Greater access to experts/specialists (nationally and internationally)

- More productive use of your time (eliminates wasted travel time) and significant travel cost savings.

- It reduces environmental impact through less travel and reduced pressure, stress and fatigue from Travel.

- Facilitating short notice meetings between individuals in distant locations thus decisions are often made more quickly. 
- Increased meeting attendance by participants who would rather be unable to hitch in.

- It gives substantial accessibility and allows geographical reach even to rural or remote locations.

- A conference session are often saved for future reference e.g. class notes are often saved and distributed via network for references by students.

\subsection{Disadvantages of Video Conferencing}

- Students who are attending video conferencing classes will lack self-discipline as they will always tend to be lazy to attend classes when at home.

- Students will never learn to make interpersonal relationships with their classmates and even with their teachers.

- India is a developing country and not everyone has access to high speed internet, so during video conferences the image and sound quality may degrade.

- The development of face to face conversation is lost and people then generally become introverted.

- The security of the videos may be compromised.

2.4. Application of Video Conferencing Video Conferencing allows access to every individual from any part of the globe to come together and interact. This helps people to easily come across varied cultures and get to know each other from the comfort of their home. Video Conferencing have also reduced cost savings on travel, earlier companies had to bear the cost of accommodation of the staff they have been sending. Video Conferencing have somehow reduced all these costs. In rural areas where Doctor can't reach immediately if we are able to make internet reach there, we still can possibly save millions of lives by just giving people some proper feedback by the doctor if it is required immediately. If some company has to train its employees then can do some remote training, this would definitely help the company save millions of dollars for not bringing the staff to be present personally.

\section{LEARNING}

We all are living in the 21 st century where we are surrounded by technology but learning is a social process, it involves growing as an individual and till we do not meet people and interact with them, we are just gaining bookish knowledge. We will not be developing the skill of communication too. Collaborative Learning helps an individual provide a good peer group for effective learning and develop as an individual. There are mainly three types of learning involved for an individual.
- Traditional Learning - Students used to go to school and colleges and attend offline classes where they used to take notes, attend seminars and laboratories. The timetable was designed by the institution

- Open Learning - Students take up open learning courses when they have some geographical restrictions of travelling or come from weaker economic sections of the society. Open Learning reduces the interaction with peers significantly low.

- Distance Learning - These programmers are also taken due to location restrictions. People in India can take up some courses in some US universities which will save them both time and money and get them the same course credibility as someone who is attending offline classes at the institute.But this also reduces peer interactions.

\section{CONCLUSION}

Video conference has its own advantages, whether a student involved in digital learning, an office person working via digital platforms or doing some educational tasks. All of them is making a revolution in the life of a person and making the things look easier than it used to be. This thing does not make a change in the print in of conceptualization. It is used in encouraging construction and its true use lies in increasing the scope of dialogue. Due to the low cost requirement in video conference technology this would revolutnize the video use. The high speed internet low accessories cost, and cheap internet connections, it is obvious that this would slowly become the basic need of the modern world. In this paper, an introduction to video conferencing and its application in distance learning was presented as an effective way of delivering subject matter in classrooms.

\section{REFERENCES}

[1] Sami Andberg (2008). Post Graduate Thesis: Video Conferencing in Distance Learning. Department Of Computer Science, University of Helsinki.

[2] Rachel Roberts (2009). Video Conferencing in Distance Learning: A New Zealand School's Perspective. Journal of Distance Learning Distance Education Association of New Zealand, Vol. 13, pp. $91-107$.

[3] Dr. Lynne .Video Conferencing in Higher Education", Institute of computer Based Learning, Heriot Watt University Edinburgh. JNT University (2007). Introduction to Video Conferencing 